\title{
28 Research Suare \\ Posterior superior alveolar nerves contribute to sensation in the anterior teeth
}

\section{Sanako Makishi}

Niigata University

Mikako Tanaka

Meirin College

Taichi Kobayashi

Niigata University

Ray Tanaka

University of Hong Kong

Takafumi Hayashi

Niigata University

Hayato Ohshima ( $\nabla$ histoman@dent.niigata-u.ac.jp )

Niigata University

\section{Research Article}

Keywords: aged, cadaver, histological techniques, three-dimensional imaging, maxillary sinus, X-ray computed tomography

Posted Date: December 18th, 2020

DOl: https://doi.org/10.21203/rs.3.rs-123843/v1

License: (c) (i) This work is licensed under a Creative Commons Attribution 4.0 International License.

Read Full License 


\section{Abstract}

There is no available data on the occurrence rate of a converged alveolar canal, the detailed threedimensional (3D) courses of alveolar canals/grooves (ACGs), or the contribution of each superior alveolar nerve to each area in the maxilla. This study aimed to clarify the 3D courses of ACGs, the relationship between ACGs and superior alveolar nerves, and the contribution of posterior superior alveolar nerves (PSANs) using computed tomography (CT) with histological analysis. During the gross anatomy course at Niigata University, we investigated nine human cadavers. All anterior and posterior ACGs converged into the common alveolar canal, which contained blood vessels and several nerve bundles surrounded by perineurium, located at the nasal floor near the pyriform aperture. Histometrical analysis clarified that $16.3 \%$ of the nerve bundles in this canal were derived from PSANs, and $67 \%$ of the bundles were dispersed while they coursed down to the nasal floor. There seems to be no relationship between the density of nerve bundles in the canal and the number of remaining anterior teeth. Data obtained from observing the detailed 3D courses of anterior and posterior ACGs, and their relationship with superior alveolar nerves, suggests that PSANs partially contribute to the nociception of the anterior teeth.

\section{Introduction}

It is of paramount importance to understand the three-dimensional (3D) relationship between the posterior superior alveolar nerves (PSANs) and the maxillary sinus in order to avoid complications such as nerve injury during antrotomy and sinus augmentation surgery (sinus floor elevation) ${ }^{1-4}$. The concept of maxillary innervation states that the anterior superior alveolar nerves (ASANs) and PSANs innervate the maxillary anterior and posterior teeth, respectively. This understanding may change if we obtain more information on the courses of ASANs and PSANs in the anterior alveolar canals which run in the anterior walls of the sinus or within sinus grooves ${ }^{5}$. Superior alveolar nerves are composed of ASANs and middle superior alveolar nerves (MSANs), which arise from the infraorbital nerve, a branch of a maxillary nerve that runs from the inferior orbital fissure to the orbita, and PSANs, which are branches of the maxillary nerve that run from the alveolar foramina into the maxillary sinus. It is thought that these superior alveolar nerves form a superior dental plexus above the apical foramina of the maxillary teeth ${ }^{6}$. The sinus has thin walls, with grooves and/or canals for the infraorbital, anterior, middle, and posterior superior alveolar nerves and vessels, and their small unnamed branches ${ }^{7}$. Regarding the topographical relationships between the superior alveolar nerves and the maxillary sinus, the branches of the PSAN run through canaliculi in the lateral wall of the sinus $(62.3 \%)$ or under the mucous membranes of the sinus $(37.8 \%)^{8}$. There is significant variation in the patterns of ASAN within the anterior face of the maxilla ${ }^{9}$. However, it is difficult to macroscopically elucidate the three-dimensional (3D) courses of the superior alveolar nerves in the maxillary sinus owing to their location in the sinus wall or under the mucous membrane of the sinus.

It is well-known that the superior alveolar nerves and vessels pass in "alveolar canals/grooves (ACGs)" 5 , although the terminology for these structures have been variously referred to as "canalis sinuosus" 6 or 
"bony canals" 10 depending on the individual researcher. Computed tomography (CT) scan data have revealed the existence of $A C G s$ 2,4,10,11 and a recent study using both $C T$ and histological observation has clarified that these structures contain alveolar nerves and vessels ${ }^{5}$. It is considered established knowledge that the maxillary nerve divides into two branches, an infraorbital nerve and PSANs. The former nerve further divides into ASANs and MSANs. Finally, three alveolar nerves innervate different tooth areas via different ACGs ${ }^{6}$ (Fig. 1a). Recently, we demonstrated that three ACGs converge into an alveolar canal located in the anterior portion of the maxilla ${ }^{5}$ (Fig. 1b). However, their occurrence rates, 3D courses of superior alveolar nerves running in these ACGs, or the exact areas innervated by each superior alveolar nerve remain to be elucidated.

To understand the 3D relationship between the maxillary sinus and the PSAN is of paramount importance in order to avoid potential complications such as nerve injury during antrostomy or sinus floor augmentation surgery ${ }^{1-4}$. The information regarding the rate of the ASAN and PSAN in the anterior alveolar canal of maxilla could elucidate the involvement of the PSAN in the nociception of the anterior teeth and their supporting tissues and may possibly modify the established theory presented in textbooks. latrogenic complications such as the injury of the PSAN, which peripherally innervates the anterior teeth, during dental implantation in the molar region might induce abnormal sensations such as anesthesia, hypesthesia, paraesthesia, and dysesthesia in the anterior region (Fig. 1c). This study aimed to clarify the 3D courses of ACGs, the relationship between ACGs and superior alveolar nerves, and the contribution of PSANs using helical CT, cone-beam CT, and $\mu$-CT combined with histological analysis.

\section{Results}

\subsection{Comparison between 3D images of superior ACGs scanned using $\mu-C T$ and histological sections}

The anterior portion of maxillae was reconstructed with bone morphometry using $\mu$-CT images included ACGs. The anterior superior ACG (ASACG) derived from the infraorbital canal/groove ran anteriorly into the anterior wall of the maxillary sinus and finally reached the bottom of the nasal cavity near the nasal notch. The posterior superior ACG (PSACG) ran in the anterosuperior direction from the lateral wall or floor of the sinus and converged with the ASACG to make a larger canal/groove at the bottom of the nasal cavity near the nasal notch (Fig. 3a, g, n; Supplementary Figs. 1-3). Next, we compared the exposed horizontal cross-sections of the 3D-reconstructed $\mu$-CT images with the H\&E-stained histological sections at the same level. These ASACGs (Fig. 3b, c, d, e, h, j, k, o, p, q, r) and PSACGs (Fig. 3e, f, i, l, m, o, $p, s)$ contained nerve bundles, arteries, and veins. The PSAN converged with the ASAN near the superior margin of the nasal notch (Fig. 3p).

\subsection{The contribution of PSAN to the nerves innervating the anterior region of maxillae}

At the point, " $x$ " and " $y$," nerve bundles, arteries, and veins were identified (Fig. 4b-d), and then divided into numerous smaller branches at the point " $\mathrm{z}$ " (Fig. 4e, f). The morphometric analyses using B/A and A-C/A showed that the rate of PSAN at the point " $x$ " was $16.3 \pm 9.8 \%$ and the converged nerves at the point " $x$ " dispersed (the rate of dispersed nerves was $67.3 \pm 20.0 \%$ ) (Fig. $4 \mathrm{~g}$ ). The remaining main converged 
nerves got thinner toward the point " $z$ " in the course from the points " $x$ " to " $z$." Finally, the main nerves emerged from the bone or remained inside, near the bottom of the nasal notch. Small dispersed nerves were recognizable at point "z." Concerning the relationship between the gross area of nerve bundles at each reference point and the number of remaining teeth in the anterior region, there was no relationship between the density of nerve bundles in the canal/groove and the number of remaining anterior teeth (Fig. 4h-j).

\section{Discussion}

Taking the images by CT before the preparation of histological analysis enabled a non-destructive morphometric analysis of ACGs located inside, or on, the bony wall of the maxillary sinus, as demonstrated by our previous study ${ }^{5}$. However, we may fail to identify the occurrence of ACGs using only CT images, since it is difficult to distinguish ACGs from bone marrow by observing one cross-section of the $3 \mathrm{D}$ reconstructed image in a sample which contains an imperfect canal structure. Moreover, the volume of bone marrow shows considerable individual and topographical differences, resulting in the failure of automatic separation between bone marrow cavities and alveolar canals, even when using bone morphometric software. Using cone-beam CT and $\mu$-CT analyses of the portion resected from a maxilla after scanning the whole cranium using helical $\mathrm{CT}$, we were able to isolate the focused objects and compare the optimal partial cross-section of 3D images of ACGs using the software with each corresponding histological section containing nerve bundles and blood vessels. Thus, the present study successfully identified the 3D structures of alveolar canals, which were previously unobservable, located in the anterior bony wall of the maxillary sinus non-destructively using the combination of $\mu-\mathrm{CT}$, histological sections, and specialized software.

The anterior superior alveolar canal running in the anterior wall of a maxillary sinus reaches to the floor of a nasal cavity along the nasal notch ${ }^{5,12}$. Serial 2D analyses with helical CT demonstrated that the ASACGs and PSACGs converged into one alveolar canal least $80 \%$ of the time, and all these nerves ran in the floor of the nasal cavity along the nasal notch ${ }^{5}$. However, it was impossible to precisely identify the occurrence of superior alveolar nerves/vessels running on the anterior wall of the maxillary sinus without a prominent canal or groove on the tomographic images, since these data were based on 2D images. The current study demonstrated that the rate of the convergence between anterior and posterior superior alveolar canals was $100 \%$ and that the converged nerves in the anterior wall of the maxillary sinus branched out into thinner nerve fibers and left the main nerve bundle in the canal/groove, running into the floor of the nasal cavity near the piriform aperture. The histological sections of the converged alveolar canal which run into the floor of the nasal cavity near the piriform aperture represent the existence of dense nerve bundles, irrespective of whether or not the cadavers had intact dentition in the anterior region. In contrast, we failed to identify thick nerve bundles in the PSACG. In the case of the inferior alveolar nerves, plural nerve bundles are separately wrapped in perineural sheaths which correspond to the innervated areas at the level of a mandibular foramen ${ }^{13,14}$. Thus, the thick nerve bundles wrapped 
with perineurium in the converged alveolar canals of the anterior wall of the sinus are likely derived from the ASANs or MSANs, whereas the thin nerve bundles likely originate from PSANs.

The anatomical textbooks note that nerves running in the ASACG, middle superior ACG (MSACG), and PSACGs never converge into one nerve bundle in the anterior superior alveolar canals; rather, conventional knowledge states that the three superior alveolar nerves innervate different areas independently (Fig. 1a). The current results demonstrated novel findings. Specifically, ASACG and PSACG consistently converge into one canal which contains anterior and posterior alveolar nerves (Fig. 1b). However, the present study failed to identify their relationship with the MSACG and nerves, since we trimmed the anterior wall of maxillary sinus and did not analyze the course of MSACGs. Thus, further studies are needed to address this issue in the future. Regarding the contribution of PSAN to the innervation of the anterior teeth region, the present study demonstrated that the rate of PSAN in the nerves running in the ASACGs was $16.3 \pm$ $9.8 \%$ and the rate of dispersed nerves was $67.3 \pm 20.0 \%$. These findings suggest that the PSAN contributes to sensation in the anterior teeth region since ASAN innervates this anterior region.

Furthermore, there appears to be no relationship between the density of nerve bundles in the canal and the number of remaining anterior teeth, indicating that the loss of teeth may not affect the sensory nerve density in the referenced area. However, this study failed to analyze this issue statistically due to the limited number of subjects and the limited variation in the remaining teeth. To date, the relationship between a superior dental plexus and remaining teeth also has not been clarified. A future study which focuses on the relationship between the 3D patterns of superior dental plexuses and remaining teeth using samples, including the alveolus of a maxilla, and increasing the sample number, could unveil the superior ACG regions (either anterior region, premolar region, or/and molar region) innervated by each ASAN, MSAN, or PSAN.

\section{Conclusions}

In the current study, the application of helical CT, cone-beam CT, $\mu$-CT, and histological analyses, combined with bone morphometric software, demonstrated a 3D relationship between nerve bundles and ACGs of the anterior wall of the maxillary sinus. Observing the detailed 3D courses of anterior and posterior alveolar canals and their relationship with superior alveolar nerves revealed that the posterior superior alveolar nerves partially contributed to nociception within the anterior teeth. In clinical dentistry, implant placement surgery in the molar region of the maxilla may elicit neurosensory disturbances of the anterior region if the posterior superior alveolar nerves are seriously injured by instrumentation, providing a clinical case where the position of the injury (molar region) and symptoms (anterior region) can be separated (Fig. 1c).

\section{Methods}

\subsection{Cone-beam $\mathrm{CT}$, helical $\mathrm{CT}$, and $\mu-\mathrm{CT}$ analyses}

Nine Japanese adult human cadavers were used for this study-five males and four females-examined during the gross anatomy courses at the Faculty of Dentistry (eight cadavers), and the Graduate School 
of Medical and Dental Sciences (one cadaver), Niigata University (2014). All dissections using cadavers were conducted in compliance with the "Preservation of Autopsy" act and the guidelines of the Ministry of Health, Labor and Welfare, and the Japanese Association of Anatomists. We obtained the informed consents of the cadavers' kin as to their use for the purpose of education and research in pursuance of the intention of the deceased. This study was reviewed by the Ethics Committee of Niigata University, approved by the President of Niigata University (28-R9-5-17), and conducted in accordance with the Declaration of Helsinki. All cadavers were routinely fixed in a formalin/phenol/alcohol solution ${ }^{15}$. The ages ranged from 79 to 96 years, with an average age of 87.9 years. Digital imaging and communication in medicine (DICOM) data obtained from the scanned heads and resected anterior portion of the maxilla (Fig. 2) using helical CT (Alexion TSX-032A/1V, Canon Medical Systems Co., Ohtawara, Japan) and conebeam CT (Auge Solio Z, Asahiroentgen Ind. Co., Ltd., Kyoto, Japan), respectively, were reconstructed into 3D images using software (Osirix, Pixmeo SARL, Bernex, Switzerland). The 3D images were compared with $\mu$-CT (Elescan, Nittetsu Elex, Tokyo, Japan) images and histological Sect. ${ }^{5}$. The scanning conditions for the cone-beam CT were as follows: field of view (FOV), $51.2 \mathrm{~mm} \times 55.0 \mathrm{~mm}$; voxel matrix, $512 \times 512 \times$ 550; voxel size, $0.1 \mathrm{~mm}$; tube voltage, $85 \mathrm{kV}$; tube current, $8 \mathrm{~mA}$; and exposure time, $17 \mathrm{~s}$. Helical CT settings were as follows: tube voltage, $120 \mathrm{kVp}$; tube current, $150 \mathrm{~mA}$; D-FOV, $220.3 \mathrm{~mm}$; C-FOV, S; and exposure time, $15.5 \mathrm{~s}$. The $\mu$-CT settings were as follows: pixel matrix, $512 \times 512 \times 256$; slice thickness, $55.143 \mu \mathrm{m}$; projection number, $900 \times 32$; magnification, 1.662; voltage $45 \mathrm{kV}$; and electrical current 101 $\mu \mathrm{A}$. The maxillae were reconstructed using a software program (TRI/3D-BON-FCS, Ratoc System Engineering, Tokyo, Japan) to evaluate the $3 \mathrm{D}$ reconstructed maxillae 5 .

\subsection{Histological procedures}

Materials were collected from the cadavers mentioned above. After the scanning using $\mu-\mathrm{CT}$, the anterior portion of maxillae were immersed in $4 \%$ paraformaldehyde in $0.1 \mathrm{M}$ phosphate buffer $(\mathrm{pH} 7.4)$ for an additional 12 hours at $4{ }^{\circ} \mathrm{C}$. Following decalcification in Morse's solution ( $10 \%$ sodium citrate and $22.5 \%$ formic acid) for 12 days at $4{ }^{\circ} \mathrm{C}$, the specimens were processed for embedding in paraffin and cut horizontally at a thickness of $4 \mu \mathrm{m}$. The serial paraffin sections were mounted on Matsunami adhesive silane (MAS)-coated glass slides (Matsunami Glass Ind., Osaka, Japan) and processed for hematoxylin and eosin (H\&E) staining ${ }^{5}$.

\subsection{The procedure for $3 D$ visualization of the ACGs}

First, the 3D images were binarized automatically and manually to extract the bone areas. Second, the binary images were inverted in black and white to visualize the structures of ACGs and bone marrow. These structures consisting of the ACGs and bone marrow, were subdivided into smaller cubes and colored according to the size of cubic volume. Subsequently, the noise corresponding to the over-selected cubes was erased, and the eroded structures were recovered manually to obtain the large continuous canal structures that were recognizable as putative ACGs. Third, to distinguish the true canals from bone marrow, these structures were modified manually by comparison with the corresponding serial $\mathrm{H} \& \mathrm{E}$ stained sections, resulting in the deletion of bone marrow cavities. The true canals were judged by the fact that the large canal structure contained nerve and vessel bundles. Next, each image of the bone 
matrix, bone marrow, and alveolar canal areas was saved onto different channels to be viewed in the monitor selectively. Finally, the bone matrix and bone marrow were rendered semitransparent, resulting in unobstructed 3D visualization of the running canals.

\subsection{Statistical analyses}

For statistical analyses, we set three reference points in the canals/grooves and named these points " $x$," " $y$," and " $z$ " on the 3D-reconstructed images (Fig. 4a). The point " $x$ " and " $z$ " of a canal/groove, where PSAN converged with ASAN, were located at the superior and inferior margin of the nasal notch, respectively. After identifying nerve bundles in the ACGs, judging from the existence of perineurium on the H\&E stained sections at the above reference points, we counted the number of nerve bundles and measured the gross area of transversely-cut nerve bundles using the Image $\mathrm{J}$ software. We selected the sections where the nerve bundles showed a round-shaped appearance. For the morphometric analyses, we calculated the rate of the diameter of PSAN at the point " $y$ " [parameter "B"] / the diameter of (ASAN + PSAN) at the point " $x$ " [parameter " $A$ "] and the parameter " $A$ " - the diameter of (ASAN + PSAN) at the point " $\mathrm{Z}$ " [parameter " $\mathrm{C}$ "] / the parameter " $\mathrm{A}$ " to clarify the rate of PSAN to the nerves innervating the anterior region of maxillae and the rate of dispersed nerves in the course from the points " $x$ " to " $z$ " using the data concerning the gross area of transversely-cut nerve bundles. Furthermore, we analyzed the relationship between the gross area of the nerve bundles at each reference point and the number of remaining teeth in the anterior region. The samples of $5 \mathrm{R}$ and $6 \mathrm{R}$ were excluded since the posterior parts containing PSAN had been out of the trimmed areas in these samples.

\section{Declarations}

\section{Acknowledgments}

The authors wish to sincerely thank those who donated their bodies to science so that anatomical research could be performed. The results from such research can potentially improve patient care and increase mankind's overall knowledge. We are grateful to Dr. Hiroko Ida-Yonemochi, Dr. Kotaro Saito, Dr. Wataru Morita, Dr. Angela Quispe-Salcedo, and Mr. Shinichi Kenmotsu for their assistance during the gross anatomy course at the Faculty of Dentistry and Graduate School of Medical and Dental Sciences, Niigata University.

\section{Author contribution}

S.M.: Contributed to design, data acquisition, analysis, and interpretation and drafted the manuscript

M.T.: Contributed to data acquisition, analysis, and interpretation and critically reviewed the manuscript

T.K.: Contributed to data acquisition and critically reviewed the manuscript

R.T.: Contributed to data acquisition and critically reviewed the manuscript

T.H.: Contributed to data acquisition and critically reviewed the manuscript 
H.O.: Contributed to the conception and design of the study, data analysis and interpretation and drafted the manuscript.

All authors gave final approval and agreed to be accountable for all aspects of the work and have agreed both to be personally accountable for their own contributions and to ensure that questions related to the accuracy or integrity of any part of the work.

\section{Additional Information}

\section{Competing Interests Statement}

The authors declare no competing interests.

\section{References}

1 Elian, N., Wallace, S., Cho, S. C., Jalbout, Z. N. \& Froum, S. Distribution of the maxillary artery as it relates to sinus floor augmentation. The International journal of oral \& maxillofacial implants 20, 784-787 (2005).

2 Ella, B. et al. Vascular connections of the lateral wall of the sinus: surgical effect in sinus augmentation. The International journal of oral \& maxillofacial implants 23, 1047-1052 (2008).

3 Solar, P. et al. Blood supply to the maxillary sinus relevant to sinus floor elevation procedures. Clin Oral Implants Res 10, 34-44 (1999).

4 Rosano, G., Taschieri, S., Gaudy, J. F., Weinstein, T. \& Del Fabbro, M. Maxillary sinus vascular anatomy and its relation to sinus lift surgery. Clin Oral Implants Res 22, 711-715, doi:10.1111/j.16000501.2010.02045.x (2011).

5 Kasahara, N. et al. The Relationships of the Maxillary Sinus With the Superior Alveolar Nerves and Vessels as Demonstrated by Cone-Beam CT Combined With mu-CT and Histological Analyses. Anat Rec (Hoboken) 299, 669-678, doi:10.1002/ar.23327 (2016).

6 Standring, S. Gray's anatomy : the anatomical basis of clinical practice. 41th edn, (Elsevier Churchill Livingstone, 2016).

7 Doig, T. N., McDonald, S. W. \& McGregor, I. A. Possible routes of spread of carcinoma of the maxillary sinus to the oral cavity. Clin Anat 11, 149-156, doi:10.1002/(SICI)10982353(1998)11:3<149::AID-CA1>3.0.C0;2-0 (1998).

8 Murakami, G. et al. The superior alveolar nerves: their topographical relationship and distribution to the maxillary sinus in human adults. Okajimas Folia Anat Jpn 70, 319-328 (1994). 
9 Robinson, S. \& Wormald, P. J. Patterns of innervation of the anterior maxilla: a cadaver study with relevance to canine fossa puncture of the maxillary sinus. The Laryngoscope 115, 1785-1788, doi:10.1097/01.mlg.0000176544.72657.a6 (2005).

10 Mardinger, O., Abba, M., Hirshberg, A. \& Schwartz-Arad, D. Prevalence, diameter and course of the maxillary intraosseous vascular canal with relation to sinus augmentation procedure: a radiographic study. International journal of oral and maxillofacial surgery 36, 735-738, doi:10.1016/j.ijom.2007.05.005 (2007).

11 Sato, I. et al. Observing the bony canal structure of the human maxillary sinus in Japanese cadavers using cone beam CT. Okajimas Folia Anat Jpn 87, 123-128 (2010).

12 Tanaka, R. et al. CT anatomy of the anterior superior alveolar nerve canal: a macroscopic and microscopic study. Oral Radiol 27, 93-97 (2011).

13 Kqiku, L., Weiglein, A. H., Pertl, C., Biblekaj, R. \& Stadtler, P. Histology and intramandibular course of the inferior alveolar nerve. Clin Oral Investig 15, 1013-1016, doi:10.1007/s00784-010-0459-x (2011).

$14 \mathrm{Hu}, \mathrm{K}$. S. et al. Branching patterns and intraosseous course of the mental nerve. J Oral Maxillofac Surg 65, 2288-2294, doi:10.1016/j.joms.2007.06.658 (2007).

15 Katsumi, Y. et al. Variation in arterial supply to the floor of the mouth and assessment of relative hemorrhage risk in implant surgery. Clin Oral Implants Res 24, 434-440, doi:10.1111/j.16000501.2011.02348.x (2013).

\section{Figures}



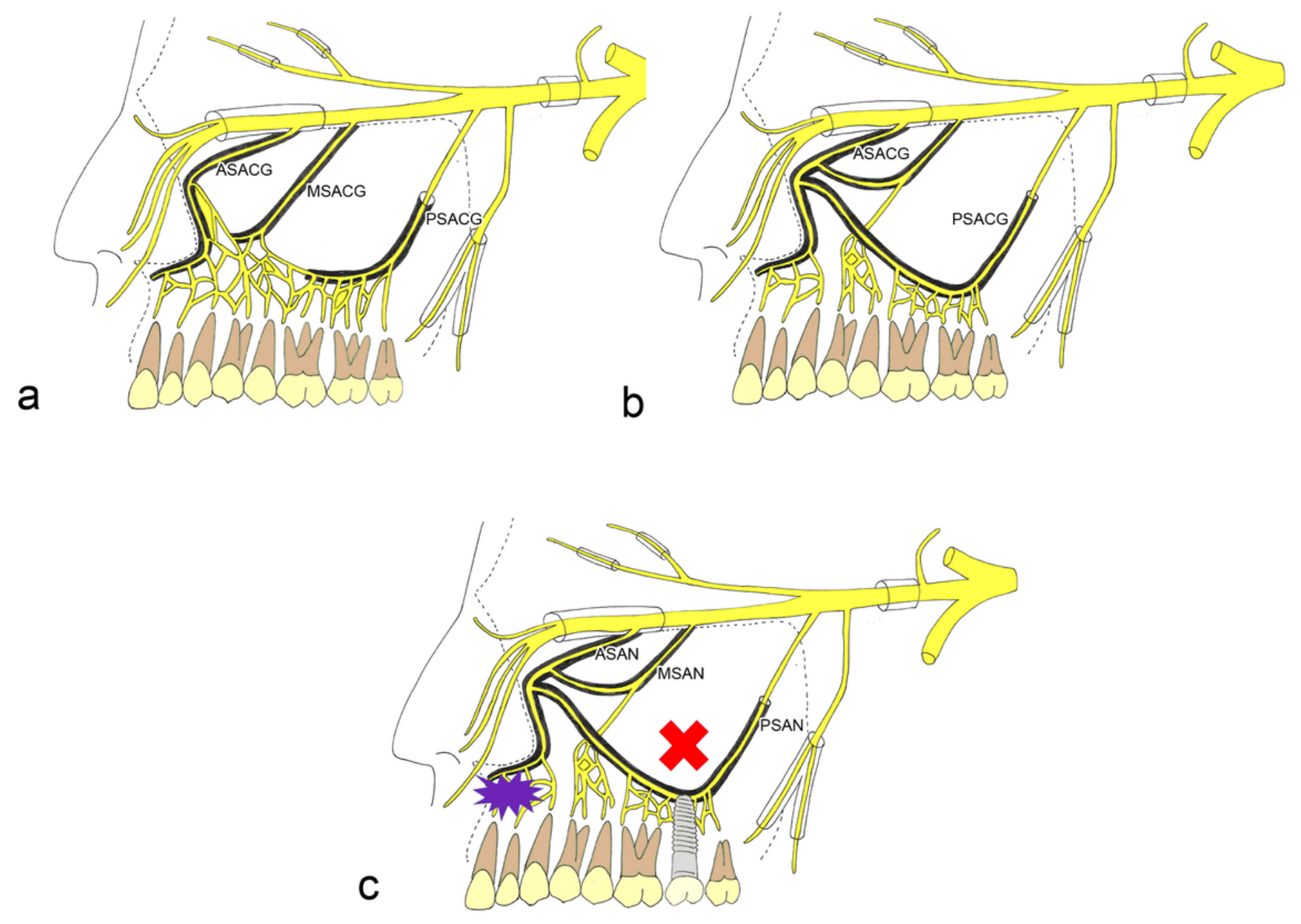

\section{Figure 1}

The comparison between conventional (a: modified from Yasuhiko Kamijo) and novel (b) concepts regarding the running course of anterior, middle, and posterior superior alveolar nerves (ASAN, MSAN, and PSAN), and their canals/grooves, and the hypothesis of a possible mechanism of complications after implant replacement (c). (a) This concept has been described in many anatomical textbooks to date. Nerves running in the anterior, middle, and posterior superior alveolar canals/grooves (ASACGs, MSACGs, and PSACGs) never converge into one nerve bundle in the anterior superior alveolar canals, and three superior alveolar nerves independently innervate different areas. (b) The current results demonstrate the novel finding that ASACGs and PSACGs consistently converge into one canal that contains the ASAN and PSAN. (c) Since the PSAN could partially contribute to the nociception of the anterior teeth, implant placement surgery in the molar region of a maxilla may elicit neurosensory disturbances of the anterior region. If the PSAN is seriously injured with instrumentation, the position of the injury (molar region) and the symptoms (anterior region) could be separated. 
a

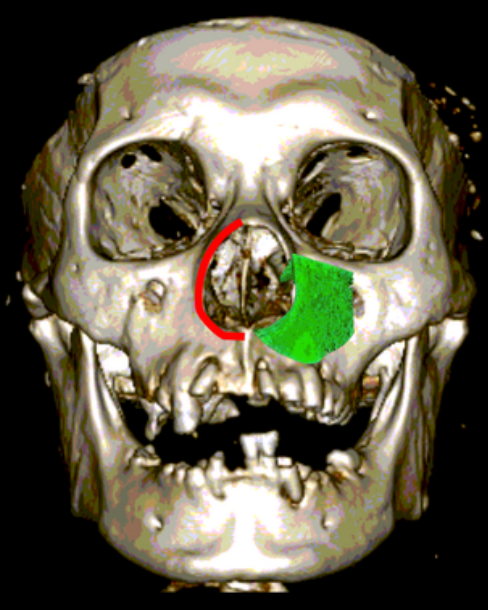

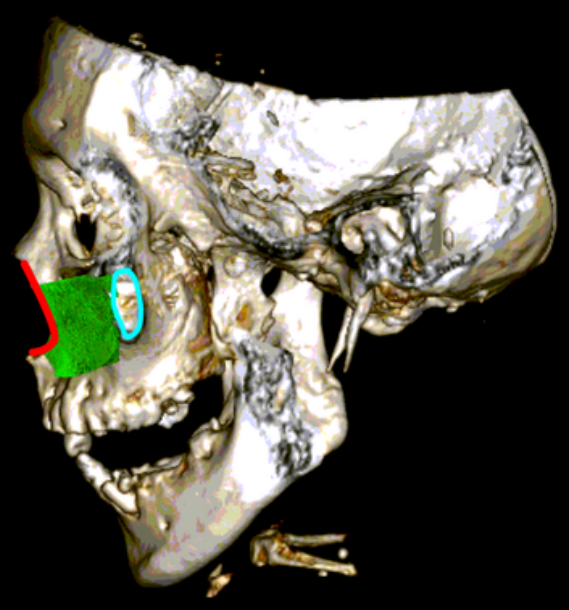

\section{Nasal notch}
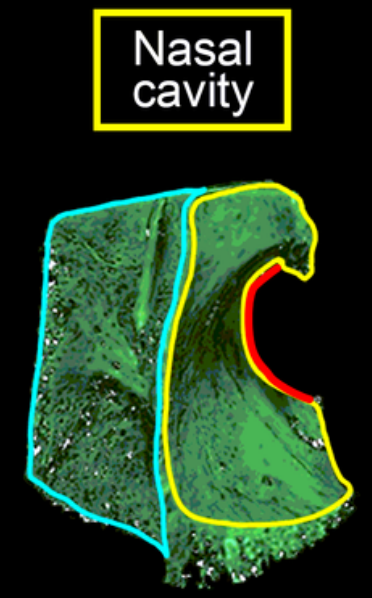

b
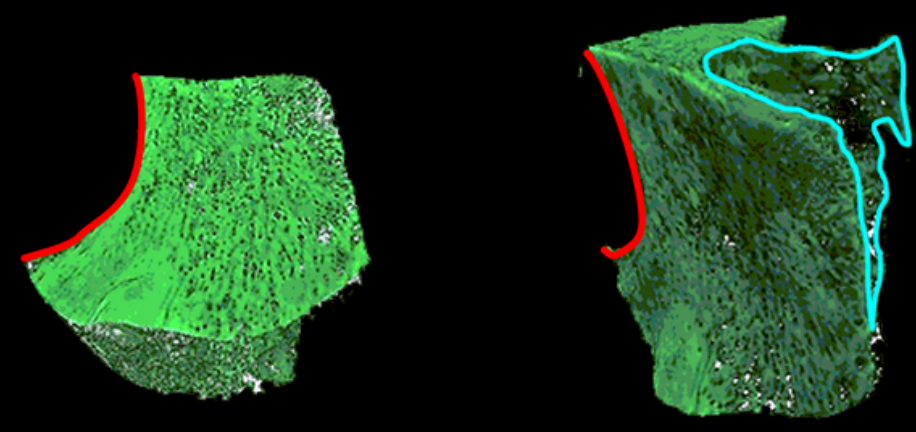

Maxillary

sinus

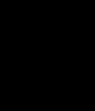

\section{Figure 2}

Three-dimensional (3D)-reconstructed images of a skull based on digital imaging and communication in medicine (DICOM) data obtained from the scanned cadavers using helical computed tomography (CT) (a) and a resected maxilla scanned using $\mu-C T$ (b). The bone matrix of the maxilla is colored green. Red lines indicated the edges of a nasal notch, and circled light blue and yellow lines demarcate the boundary of the maxillary sinus and nasal cavity, respectively. 


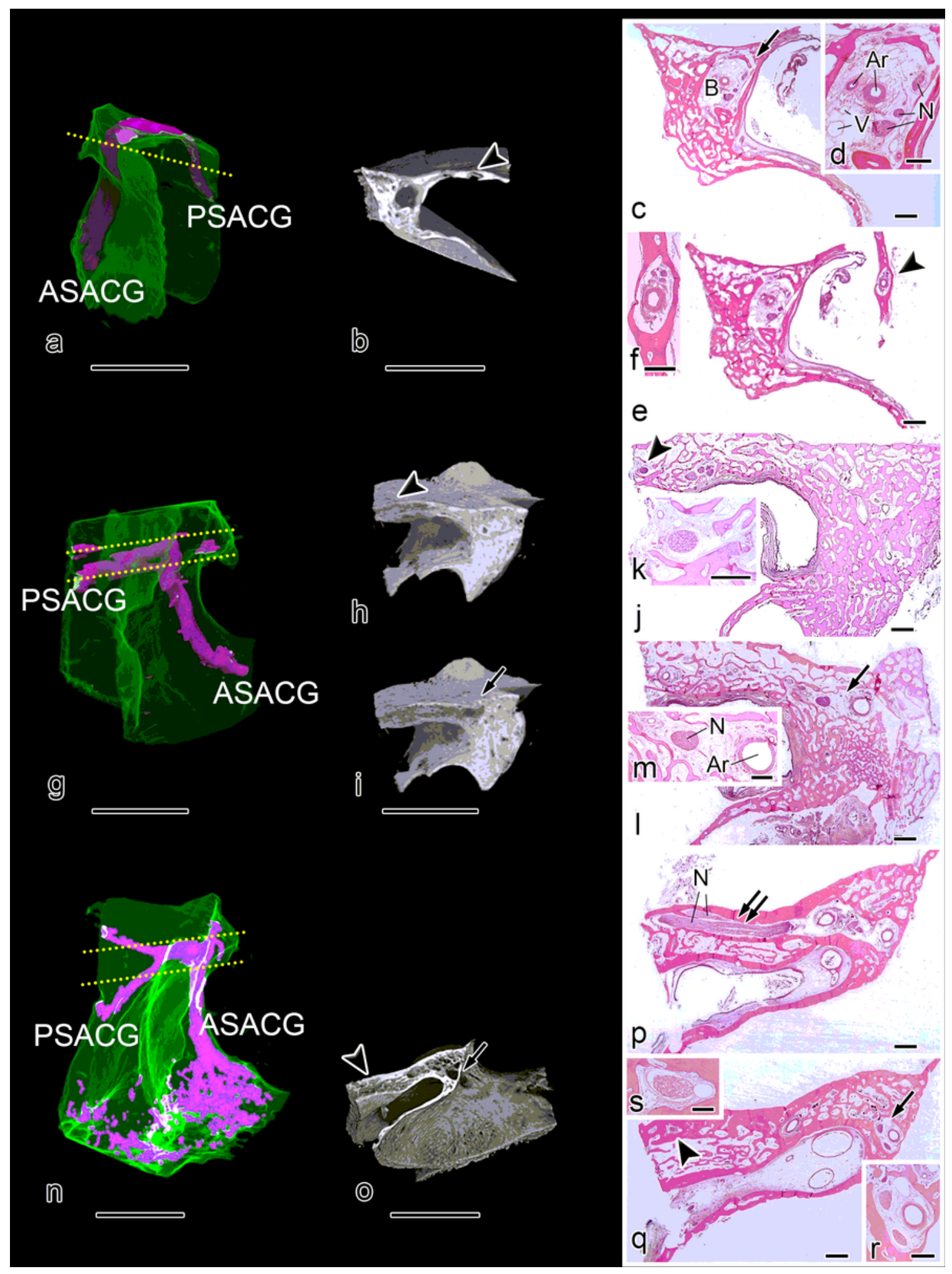

Figure 3

3D-reconstructed images of trimmed maxillae using $\mu-C T(a, b, g, h, i, n, 0)$ and histological sections $(c, d$, $e, f, j, k, l, m, p, q, r, s)$. Using a software program (TRI/3D-BON-FCS, Ratoc System Engineering, Tokyo, Japan), the bone and canals/grooves are converted into colored green and purple, and their opacities are lower and higher, respectively. The trimmed 3D-reconstructed images of superior alveolar canals/grooves (ACGs) $(b, h, i, o)$ correspond to each histological section (e, j, l, q). Yellow dotted lines indicate their 
trimmed surfaces. Note that PSACG converges together with ASACG at the middle edge of a nasal notch. ASACG, anterior superior alveolar canal/groove; PSACG, posterior superior alveolar canal/groove; N, nerves; Ar, arteries; V, veins; arrows; ASACGs, arrowheads; PSACGs, double arrows; converged anterior and posterior superior alveolar nerves running together horizontally in ASACG. Scale bars $=10 \mathrm{~mm}(\mathrm{a}, \mathrm{b}, \mathrm{g}, \mathrm{h}, \mathrm{i}$, $n, 0), 1000 \mu m(c, e, j, l, p, q), 500 \mu m(d, f, k, m, s, r)$.
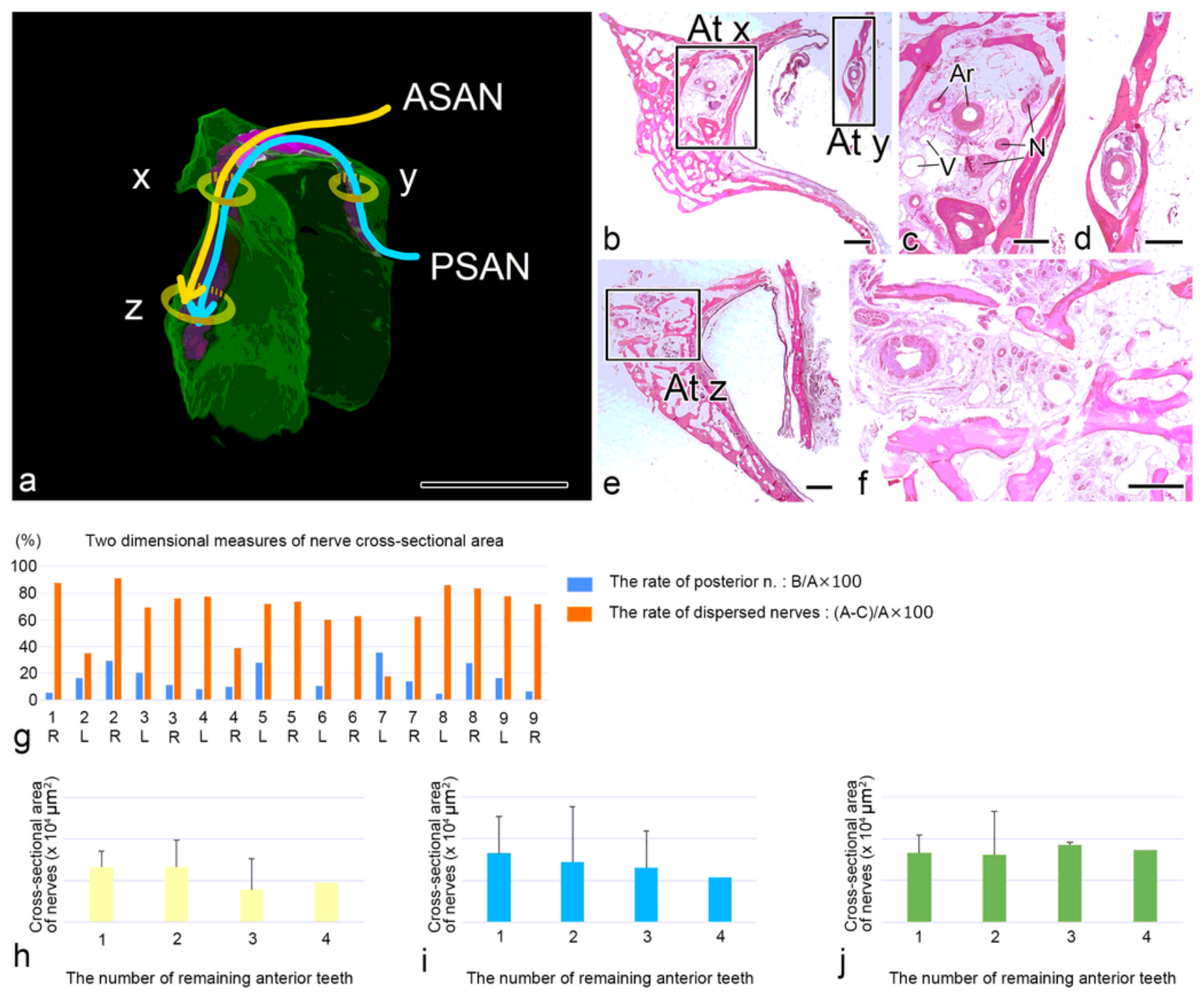

\section{Figure 4}

3D-reconstructed images of trimmed maxillae using $\mu-C T$ (a), histological sections cut at the levels of points $x, y(b, c, d)$ and $z(e, f)$, the rate of PSAN in the nerve bundles at point and dispersed nerves in ASACG during points $x$ to $z(g)$, and the cross-sectional areas of nerves at points $x(h)$ and $y(i)$ and dispersed nerves during points $x$ to $z(j)$ in different numbers of remaining anterior teeth. (a) Three points $(x, y$, and $z)$ in the courses of ASAN and PSAN running inside of ASACG or PSACG are set for measuring the areas of sectioned nerves. (b-f) PSAN is observed alone and shows a circle-shaped appearance at the point of $y(d)$. At $x$, ASAN and PSAN converge together in one ASACG (c) that disperses during the points $x$ to $z$ (f). (g) On average, the rate of PSAN amounts to $16 \%$ of all nerve bundles at point $x$. In $5 R$ and $6 R$, posterior parts, including PSAN, are out of its trimmed area so that they are missing in this chart. The rate 
of dispersed nerves amounts to $67 \%$ of the main nerve bundle during points $x$ to $z$. (h-j) There are no statistical differences between the sectional-areas of nerves at points $x(h)$ and $y$ (i) and dispersed nerves

during points $x$ to $z(j)$ and remaining anterior teeth. ASACG, anterior superior alveolar canal/groove;

PSACG, posterior superior alveolar canal/groove; N, nerves; Ar, arteries; $\mathrm{V}$, veins. Scale bars $=10 \mathrm{~mm}(\mathrm{a})$, $1000 \mu \mathrm{m}(\mathrm{b}, \mathrm{e}), 500 \mu \mathrm{m}(\mathrm{c}, \mathrm{d}, \mathrm{f})$.

\section{Supplementary Files}

This is a list of supplementary files associated with this preprint. Click to download.

- Appendix.docx

- Supplementary.docx 